\title{
Surface Roughness Imaging of Currents Shows Divergence and Strain in the Wind Direction
}

\author{
NiCOLAS RASCLE \\ Laboratoire d'Océanographie Spatiale, Institut Francais de Recherche pour l'Exploitation de la Mer, Plouzané, France, \\ and Nansen-Tutu Center, Department of Oceanography, University of Cape Town, Rondebosch, South Africa \\ BERTRAND CHAPRON \\ Laboratoire d'Océanographie Spatiale, Institut Francais de Recherche pour l'Exploitation de la Mer, Plouzané, France \\ AURÉLIEN PONTE \\ Laboratoire de Physique des Océans, Institut Francais de Recherche pour l'Exploitation de la Mer, Plouzané, France \\ FABRICE ARDHUIN \\ Laboratoire d'Océanographie Spatiale, Institut Francais de Recherche pour l'Exploitation de la Mer, Plouzané, France \\ PATRICE KLEIN \\ Laboratoire de Physique des Océans, Institut Francais de Recherche pour l'Exploitation de la Mer, Plouzané, France
}

(Manuscript received 16 December 2013, in final form 25 April 2014)

\begin{abstract}
Images of sea surface roughness-for example, obtained by synthetic aperture radars (SAR) or by radiometers viewing areas in and around the sun glitter-at times provide clear observations of meso- and submesoscale oceanic features. Interacting with the surface wind waves, particular deformation properties of surface currents are responsible for those manifestations. Ignoring other sources of surface roughness variations, the authors limit their discussion to the mean square slope (mss) variability. This study confirms that vortical currents and currents with shear in the wind direction shall not be expressed in surface roughness images. Only divergent currents or currents with no divergence but strained in the wind direction can exhibit surface roughness signatures. More specifically, nondivergent currents might be traced with a $45^{\circ}$ sensitivity to the wind direction. A simple method is proposed in order to interpret high-resolution roughness images, where roughness variations are proportional to $\partial u / \partial x+\alpha \partial v / \partial y$, a linear combination of the along-wind and crosswind current gradients. The polarization parameter $\alpha$ depends upon the sensor look direction and the directional properties of the surface waves selected by the sensor. The use of multiple look directions or possible acquisitions with different wind directions shall thus help to retrieve surface currents from surface roughness observations.
\end{abstract}

\section{Introduction}

Images of sea surface roughness are routinely obtained using active or passive sensors-such as microwave radars including synthetic aperture radar (SAR) (e.g., Beal et al.

Corresponding author address: Nicolas Rascle, Laboratoire d'Océanographie Spatiale, IFREMER, ZI Pointe du Diable, 29280 Plouzané, France.

E-mail: nicolas.rascle@ifremer.fr
1981) or optical radiometers viewing areas in and around the sun glitter (e.g., Apel et al. 1975) - that operate at different wavelengths, polarizations, and viewing angles. Under low to moderate wind conditions, those images provide clear observations of a wide range of oceanic phenomena at scales of about 1 to $30 \mathrm{~km}$, including internal waves, mesoscale, and submesoscale features such as fronts, filaments, and spiraling eddies (e.g., Fu and Holt 1983; Alpers 1985). Figure 1 shows an example of such an image. Because surface currents can strongly 


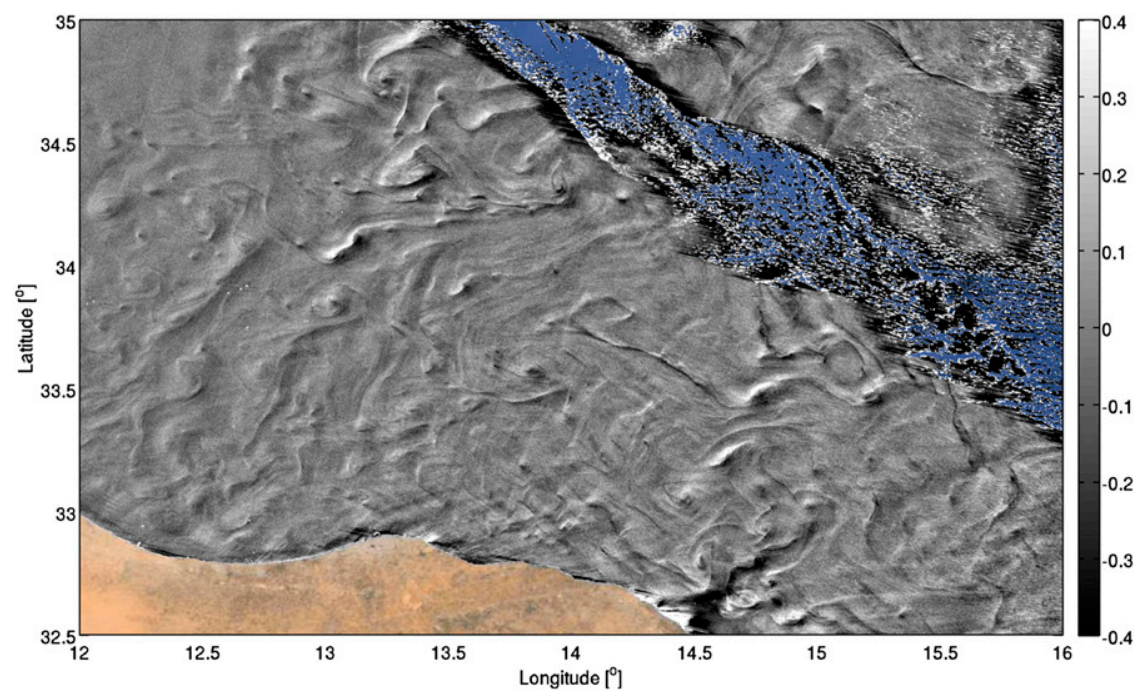

FIG. 1. Surface roughness in the Libyan region of the Mediterranean Sea observed by optical sensor in the sun glitter. Clouds are colored in blue and land is colored in brown. This image was obtained on 3 March 2010 from the 900-nm radiance measured by the Medium Resolution Imaging Spectrometer (MERIS) (Huot et al. 2001) onboard Environmental Satellite (Envisat), in the area within $20^{\circ}$ of the specular sun glint. This image has a resolution of $250 \mathrm{~m}$ and has been high-pass filtered at $25 \mathrm{~km}$.

affect small-scale (capillaries and short gravity) waves under moderate wind conditions, it is possible to track and quantify current gradients (Kudryavtsev et al. 2005; Johannessen et al. 2005).

Spurred partly by improvements in computing power, numerical models have recently confirmed the importance of ocean features at the submesoscale for exchanges within the ocean and between the ocean and the atmosphere (e.g., Fox-Kemper et al. 2011; Lévy et al. 2012; Ferrari 2011). There is however a lack of systematic observations at these scales. Efforts to remedy this situation are under way, as illustrated by the upcoming Surface Water and Ocean Topography (SWOT) satellite mission (Fu et al. 2012), but at the moment observations at these scales come mainly from images of infrared-based sea surface temperature and ocean color reflectance. The use of surface roughness images has been largely overlooked in that context, whereas with large footprints and high-resolution capabilities, surface roughness images can already be valuable to further improve qualitative and quantitative understandings of upper-ocean submesoscale processes.

Surface roughness variations in sun glitter images are controlled by specularly reflected sunlight (Cox and Munk 1954) and related to changes of wave slope distribution. The latter mostly correspond to variations of mean square slope (mss) (Kudryavtsev et al. 2012b). For radar images, the interpretation is more complex as radar backscatter intensity is affected by the mss but also by scattering effects and geometrical properties of the surface (Kudryavtsev et al. 2005). Details on how to estimate mss from glitter brightness or from radar backscatter are beyond the scope of the present paper, and the reader is referred to Kudryavtsev et al. (2012b). For simplicity in this paper, we will assume an unequivocal relationship between surface roughness and mss, which is an adequate assumption at least for optical observations.

Local variations of surface roughness have been attributed to three main processes: direct wave-current interactions (e.g., Johannessen et al. 1996), attenuation by surfactants accumulated in current convergence zones (e.g., Espedal et al. 1998), and varying wind field resulting from transformations of the atmospheric boundary layer across sea surface temperature fronts (e.g., Beal et al. 1997). Surface slicks have specific roughness signatures and mainly occur in coastal waters under light wind conditions (e.g., McWilliams et al. 2009). In this paper, we do not consider the effects of surfactants. Atmospheric boundary layer adjustments depend on the atmosphere stability induced by changes in the sea surface temperature. ${ }^{1}$ Variations of atmospheric stability seemingly occur at scales larger than

\footnotetext{
${ }^{1}$ Under moderate wind conditions, there is also a local coupling between the atmosphere flow and surface roughness. This effect shall follow roughness variations and further enhance them, as the form drag scaled by the squared friction velocity is directly linked to the mss (Plant 1982).
} 


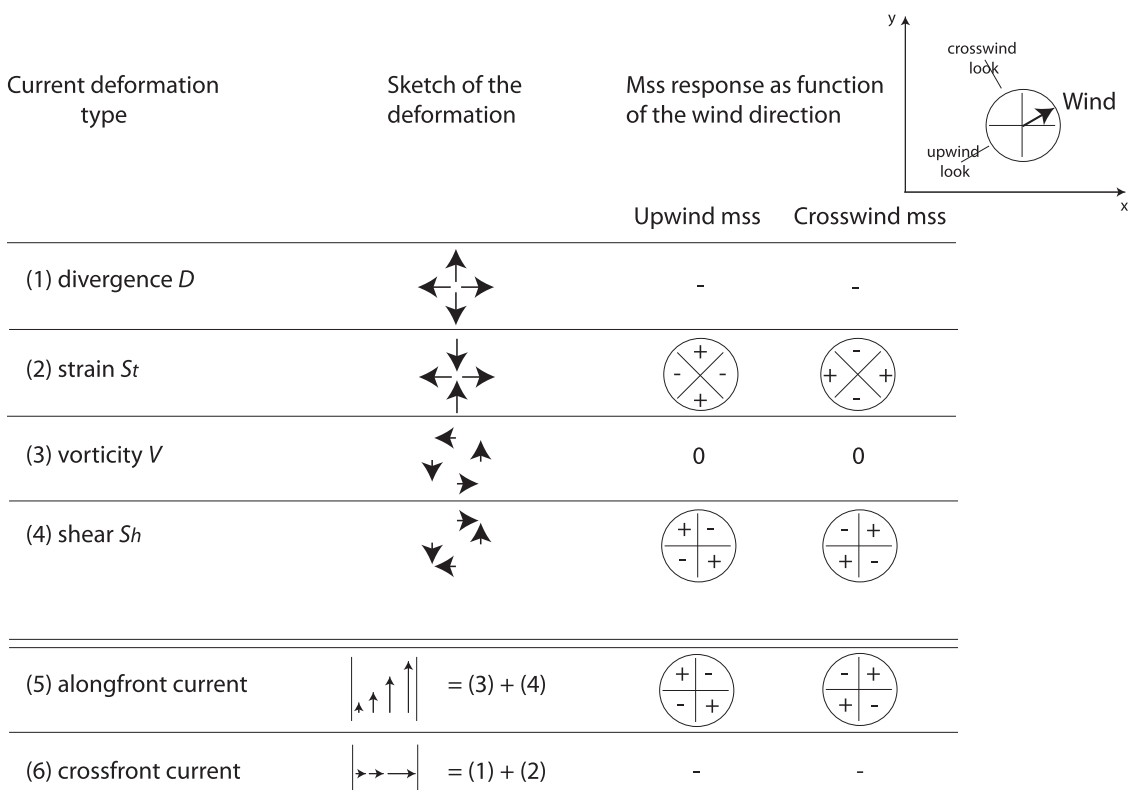

FIG. 2. Sketches (1)-(4) are the deformation tensor decomposition into divergence, strain in the $x$ direction, vorticity, and shear in the $x$ direction. The typical sign of the upwind and crosswind (for a broad wave spectrum see section $3 \mathrm{~b}$ or without roughness generation by breaking see section 3d) mss response to such a current is also shown as a function of the wind direction. (5)-(6) Simple current features such as alongfront and cross-front currents are shown with their decomposition on such deformation basis. Note that here the wind is no longer along the $x$ axis so that we replace the $x$ and $y$ projections of the mss by the upwind and crosswind projections.

those shown in Fig. 1 (see, e.g., Kudryavtsev et al. 2005, their section 4), therefore we do not consider these effects either.

Thus, not considering the processes associated with surfactants and atmospheric stability, the primary process that helps imaging the underlying flow dynamics is governed by interactions between surface wind waves and surface currents. Accordingly, questions arise. What information on ocean turbulence at meso- and submesoscale is contained in surface roughness variations? Surface current gradients can be described with four types of deformations: divergence, vorticity, strain, and shear $^{2}$ (Fig. 2, sketches 1 to 4). Which type of current deformation is mostly visible in roughness images? Can we develop a method that inverts surface roughness into current information?

The surface roughness images that are most easily interpreted are those depicting currents induced by bottom topography or by internal waves (e.g., Alpers 1985), where clear patterns of convergence/divergence

\footnotetext{
${ }^{2}$ In the literature, "strain" is also called "stretch" (e.g., Okubo 1970) or "normal strain" (e.g., Hua 1994) and "shear" is also called "shear strain."
}

of surface currents are detected as bright/dark contrasts. More generally, an individual surface wave traveling over a varying current might be deflected by the gradient of the cross-propagation current (sometimes inadequately called current shear, Fig. 2, sketch 5, if we suppose that the wave travels in the $x$ direction), but the gradient of the along-propagation current (sometimes inadequately called divergence, Fig. 2, sketch 6) can more strongly alter the steepness properties (e.g., Phillips 1984). As such, remote sensing experts often refer the current deformation as current divergence, implicitly assuming that it refers to the gradient of the alongpropagation current (Phillips 1984; Alpers and Hennings 1984).

Based on the conservation of action (e.g., Phillips 1984), modulation not only of an individual wave but of a full spectrum of wind waves can be determined. Such spectrum with waves propagating in different directions is modulated by different current deformation types, including current divergence but also current shears. Dulov and Kudryavtsev (1990) demonstrated the latter effect by considering fluctuations of wave breaking intensity. Yet, subsequent works reported the effect of current shear to be negligible compared to that of current divergence (e.g., Jansen et al. 1998). As such, it has 
become customary to assume that divergence was the dominant deformation type (e.g., Johannessen et al. 2005; Kudryavtsev et al. 2005, 2012a). This is indeed a good approximation for currents with significant divergence, as those induced by bottom topography (Alpers and Hennings 1984), by internal waves, or along sharp density fronts (Jansen et al. 1998). On the other hand, for meso- and submesoscale turbulence, currents are constrained by Earth rotation and consequently exhibit weak divergence and larger shears. Thus, it might be somehow misleading to immediately assume that current divergence is into play whenever effects of current deformation are explored.

In this paper, we wish to clarify the interpretation of surface roughness variations in terms of current deformation. In section 2, we recall the modulation of waves by currents, extend it from a single wave to a spectrum symmetrical about the wind direction, and calculate resulting mss variations. Out of the four types of surface current deformations, two can exhibit a roughness signature, the divergence and the strain in the wind direction, whereas the vorticity and the shear in the wind direction have no signature. The wind direction and the sensor look direction are irrelevant for divergence detection. This is not the case for strain detection. In section 3 , we describe the roughness responses to divergence and strain for different spectral angular spreads of wind waves. This provides a simple method to interpret roughness images, depending on the wave directional spread and the sensor look direction. In section 4, results are illustrated using a forward numerical simulation including mss variations. Conclusions and perspectives are finally drawn in section 5 .

\section{Surface roughness and current deformation types}

\section{a. Surface waves in currents}

In the presence of a current, the conservation of wave action $N(\mathbf{x}, \mathbf{k}, t)$ reads as (e.g., Phillips 1984)

$$
\frac{\partial N}{\partial t}+\left(c_{g i}+u_{i}\right) \frac{\partial N}{\partial x_{i}}=k_{j} \frac{\partial u_{j}}{\partial x_{i}} \frac{\partial N}{\partial k_{i}}+\frac{S}{\omega},
$$

where $\mathbf{c}_{\mathbf{g}}$ is the group velocity, $\mathbf{u}$ is the current, $\mathbf{x}$ is the horizontal position, $\mathbf{k}$ is the wavenumber, $\omega$ is the intrinsic frequency, and $S$ is the energy source that contains input from the wind, dissipation, and wave-wave nonlinear interactions. Repeated indices $i, j=1$ to 2 indicate summation over horizontal components.
Following a relaxation approach (e.g., Keller and Wright 1975; Hughes 1978; Alpers and Hennings 1984), the action is written $N(\mathbf{x}, \mathbf{k}, t)=N_{0}(\mathbf{k})+\tilde{N}(\mathbf{x}, \mathbf{k}, t)$, where it is supposed to experience a small disturbance $\tilde{N}$ with respect to a background value $N_{0}$ corresponding to the state undisturbed by currents. Sources rapidly restore equilibrium and are represented by a linear relaxation $S / \omega=-\tilde{N} / \tau_{c}$, where the time scale $\tau_{c}(\mathbf{k})$ depends on the wavenumber. Assuming that the typical length scale $L$ of the current is larger than the relaxation length scale $l_{r}=\tau_{c}\left|\mathbf{c}_{\mathbf{g}}\right|$, the advection term on the left-hand side of (1) can be ignored. In a steady state, the action anomaly $\tilde{N}$ due to current variations then is

$$
\tilde{N}(\mathbf{x}, \mathbf{k})=\tau_{c} k_{j} \frac{\partial u_{j}}{\partial x_{i}} \frac{\partial N_{0}}{\partial k_{i}} .
$$

And in polar coordinates

$$
\begin{aligned}
\tilde{N}(\mathbf{x}, k, \phi)= & \tau_{c} N_{0}\left\{\frac{\partial u}{\partial x}\left[\cos ^{2}(\phi) m_{k}-\cos (\phi) \sin (\phi) m_{\phi}\right]\right. \\
& +\frac{\partial u}{\partial y}\left[\cos (\phi) \sin (\phi) m_{k}+\cos ^{2}(\phi) m_{\phi}\right] \\
& +\frac{\partial v}{\partial x}\left[\cos (\phi) \sin (\phi) m_{k}-\sin ^{2}(\phi) m_{\phi}\right] \\
& \left.+\frac{\partial v}{\partial y}\left[\sin ^{2}(\phi) m_{k}+\cos (\phi) \sin (\phi) m_{\phi}\right]\right\},
\end{aligned}
$$

where $k$ and $\phi$ are the wavenumber magnitude and direction, where $m_{k}=\partial \ln N_{0} / \partial \ln k$ and $m_{\phi}=\partial \ln N_{0} / \partial \phi$, and where notations $x, y$ and $u, v$ are used in place of $x_{1}$, $x_{2}$ and $u_{1}, u_{2}$.

\section{b. Surface roughness changes}

As stated in the introduction, we suppose that the detected variations of surface roughness directly relate to local mss variations. From the wave action anomaly $\tilde{N}$, the mss anomalies are

$$
\begin{aligned}
& \widetilde{\mathrm{mss}}_{t}(\mathbf{x})=\int_{k} \int_{\phi} \omega^{-1} k \tilde{N} k^{2} d k k d \phi, \\
& \widetilde{\mathrm{mss}}_{x}(\mathbf{x})=\int_{k} \int_{\phi} \omega^{-1} k \tilde{N} k^{2} \cos ^{2} \phi d k k d \phi, \\
& \widetilde{\mathrm{mss}}_{y}(\mathbf{x})=\int_{k} \int_{\phi} \omega^{-1} k \tilde{N} k^{2} \sin ^{2} \phi d k k d \phi,
\end{aligned}
$$

where $\widetilde{\mathrm{mss}_{x}}$ and $\widetilde{\mathrm{mss}_{y}}$ are the $x$ and $y$ projection of the total mss anomaly $\widetilde{\mathrm{mss}}_{t}$. 
The wavenumber interval where the integration has to be performed is sensor dependent. For instance, at high zenith incidence angles, SARs in the $\mathrm{C}$ band are rather sensitive to scattering by waves of the order of $0.1-\mathrm{m}$ wavelength. On the contrary, sun glitters involve specular reflections by waves from the whole spectrum.

Additionally, a sensor might not be sensitive to the total slope distribution $\mathrm{mss}_{t}$ but rather to the distribution of slopes in the azimuthal look direction of the sensor (e.g., mss $_{x}$ if the sensor looks in the $x$ direction). That sensitivity is not well established yet \{see Kudryavtsev et al. [2005, their (10)] for the SAR and see Kudryavtsev et al. [2012b, their (6)] for the sun glitter\}. Awaiting further improvements, one might consider that a sensor detects a weighted average of the total mss and the mss projection in the look direction.

\section{c. Current tensor decomposition}

To ease the analysis, the deformation tensor $\partial u_{j} / \partial x_{i}$ is separated in divergence $D$, vorticity $V$, strain $S_{t}$, and shear $S_{h}$ :

$$
\begin{gathered}
D=\frac{\partial u}{\partial x}+\frac{\partial v}{\partial y}, \quad S_{t}=\frac{\partial u}{\partial x}-\frac{\partial v}{\partial y}, \\
V=\frac{\partial v}{\partial x}-\frac{\partial u}{\partial y}, \quad S_{h}=\frac{\partial v}{\partial x}+\frac{\partial u}{\partial y}, \quad \text { and } \\
{\left[\begin{array}{cc}
\frac{\partial u}{\partial x} & \frac{\partial u}{\partial y} \\
\frac{\partial v}{\partial x} & \frac{\partial v}{\partial y}
\end{array}\right]=\frac{1}{2}\left[\begin{array}{cc}
D+S_{t} & -V+S_{h} \\
V+S_{h} & D-S_{t}
\end{array}\right] .} \\
\text { That decomposition is sketched in Fig. 2. } \\
\text { The action anomaly in }(3) \text { is then rewritten } \\
\tilde{N}(\mathbf{x}, k, \phi)=\frac{\tau_{c} N_{0}}{2}\left\{D m_{k}-V m_{\phi}+S_{t}\left[\cos (2 \phi) m_{k}\right.\right. \\
\left.\left.-\sin (2 \phi) m_{\phi}\right]+S_{h}\left[\sin (2 \phi) m_{k}+\cos (2 \phi) m_{\phi}\right]\right\} .
\end{gathered}
$$

\section{d. Wind-wave spectral symmetry}

The proposed decomposition is valid in any direction. Yet, wind-wave spectral energy is concentrated in the wind direction. Without loss of generality, we will assume that the wind direction is aligned with the $x$ axis.

We will also assume that the angular spread of the wave spectrum is symmetrical about the wind direction. The wave spectrum is then an even function of $\phi$, and the same property is transferred to the relaxation time $\tau_{c}(k, \phi)$. Note that $m_{k} N_{0}$ is even whereas $m_{\phi} N_{0}$ is odd.
Any integral over the wave directions $\phi$ of an odd function will cancel out in the mss response (Kudryavtsev et al. 2005). Consequently, this results in the following:

- The mss (any mss, i.e., $\mathrm{mss}_{x}, \mathrm{mss}_{y}$, or $\mathrm{mss}_{t}$ ) response to vortical currents $(V)$ is zero, since $m_{\phi}$ is an odd function of $\phi$. This physically means that mss anomaly due to the modulation of waves traveling in the direction $\phi$ is exactly compensated by that of waves traveling in the direction $-\phi$.

- The mss response to sheared currents $\left(S_{h}\right)$ is also zero, since $\sin (2 \phi) m_{k}+\cos (2 \phi) m_{\phi}$ is also odd.

- The mss response to divergent currents $(D)$ is nonzero, since $m_{k}$ is even.

- The mss response to strained currents $\left(S_{t}\right)$ is nonzero, since $\cos (2 \phi) m_{k}-\sin (2 \phi) m_{\phi}$ is also even.

Vortical and sheared currents will thus not appear as surface roughness variations. Only currents with divergence or with strain in the wind direction will have a signature on the mss.

These results are translated onto the canonical base of the tensor [(3)] as follows: $\partial u / \partial y$ and $\partial v / \partial x$ will have no signature in terms of mss, while $\partial u / \partial x$ and $\partial v / \partial y$ will have a nonzero signature. A polarization in the wind direction (i.e., the nonzero response to strain in that direction) appears because the responses to $\partial u / \partial x$ and to $\partial v / \partial y$ are generally not equal. This will be quantified in section $3 \mathrm{a}$ by defining a polarization index.

\section{e. Wind direction dependency}

Divergence $D$ and vorticity $V$ of the currents are independent of the axis orientation. This is not the case for the strain $S_{t}$ and shear $S_{h}$. For a change of coordinates, if $\theta$ is the rotation to the new coordinates, ${ }^{3}$ the strain and shear are not conserved and transform as follows:

$$
\left[\begin{array}{l}
S_{t}^{\prime} \\
S_{h}^{\prime}
\end{array}\right]=\left[\begin{array}{cc}
\cos 2 \theta & -\sin 2 \theta \\
\sin 2 \theta & \cos 2 \theta
\end{array}\right]\left[\begin{array}{l}
S_{t} \\
S_{h}
\end{array}\right]
$$

This explicitly shows that the strain in one direction is related to shear in a direction rotated by $45^{\circ}$. The

${ }^{3}$ For a change of coordinates, $\Theta=\left[\begin{array}{cc}\cos \theta & -\sin \theta \\ \sin \theta & \cos \theta\end{array}\right]$ is the $\theta$ rotation tensor to the new coordinates. A deformation tensor $\mathbf{T}$ changes in the new coordinates to $\mathbf{T}^{\prime}=\boldsymbol{\Theta}^{t} \mathbf{T} \boldsymbol{\Theta}$. The divergence tensor $\mathbf{D}=\left[\begin{array}{cc}D & 0 \\ 0 & D\end{array}\right]$ and the vorticity tensor $\mathbf{V}=\left[\begin{array}{cc}0 & -V \\ V & 0\end{array}\right]$ of the current are then conserved, that is, $D^{\prime}=D$ and $V^{\prime}=V$, because they commute with $\boldsymbol{\Theta}$. On the contrary, the strain tensor $\mathbf{S}_{\mathbf{t}}=$ $\left[\begin{array}{cc}S_{t} & 0 \\ 0 & -S_{t}\end{array}\right]$ and the shear tensor $\mathbf{S}_{\mathbf{h}}=\left[\begin{array}{cc}0 & S_{h} \\ S_{h} & 0\end{array}\right]$ are not conserved. 
two orthogonal directions for which $S_{h}^{\prime}=0$ define the major and minor axes of the deformation (e.g., Kundu 1990, p. 60).

To illustrate our purpose, consider an infinite front with alongfront current $[u=0 ; v=v(x)$; see sketch 5 in Fig. 2]. This current has no divergence and no strain in the $x$ direction, $D=S_{t}=0$, but has nonzero vorticity and nonzero shear in the $x$ direction, $V=S_{h}=\partial v / \partial x$. If the wind blows at an angle $\theta$, the strain in the wind direction reads $S_{t}^{\prime}=-\sin (2 \theta) S_{h}$ according to (8). Such a front will therefore not appear in roughness images when the wind blows in the north-south or east-west directions. But, if the wind blows obliquely (the maximum being at $45^{\circ}$ angle), the current will have a nonzero strain in the wind direction and will thus appear in roughness images.

In summary, divergent currents with no strain and no shear will appear in surface roughness images independently of the wind direction, with a roughness anomaly independent of the wind direction (see Fig. 2). For nondivergent currents, the relative direction of the wind is crucial. Only currents strained in the wind direction will exhibit an mss signature, while currents strained $45^{\circ}$ to the wind will not appear. If the wind turns by $90^{\circ}$, the signature of strained currents will further change sign.

\section{Toward a practical interpretation of surface roughness variations}

The analysis presented so far demonstrates that some current deformations appear in surface roughness images. Analysis of such images in the sun glitter spot (Cox and Munk 1954), or use of ancillary sources (e.g., models or other satellite data), can provide additional information about the wind speed and direction. It is then of interest what information we can extract about the current deformation field when we combine all this information.

\section{a. Polarization index}

In this section the wind is set again in the $x$ direction. Because the mss responses to $\partial u / \partial x$ and to $\partial v / \partial y$ are generally not equal, we introduce a new parameter, the polarization index, to quantify the relative contribution of each current gradient. The total mss response to current can be rewritten from (3) and (4) as

$$
\widetilde{\mathrm{mss}_{t}}=\left[\frac{\partial u}{\partial x}+\alpha \frac{\partial v}{\partial y}\right] \times \int_{k} \int_{\phi} \omega^{-1} \tau_{c} k^{3} N_{0}\left[\cos ^{2}(\phi) m_{k}-\cos (\phi) \sin (\phi) m_{\phi}\right] d k k d \phi,
$$

where we define the polarization index as

$$
\alpha_{t}=\frac{\int_{k} \int_{\phi} \omega^{-1} \tau_{c} k^{3} N_{0}\left[\sin ^{2}(\phi) m_{k}+\cos (\phi) \sin (\phi) m_{\phi}\right] d k k d \phi}{\int_{k} \int_{\phi} \omega^{-1} \tau_{c} k^{3} N_{0}\left[\cos ^{2}(\phi) m_{k}-\cos (\phi) \sin (\phi) m_{\phi}\right] d k k d \phi} .
$$

We can define similarly the polarization indexes $\alpha_{x}$ and $\alpha_{y}$ for the $x$ and $y$ components of the mss.

Note that a combination $\partial u / \partial x+\alpha \partial v / \partial y$ corresponds to a divergence and strain combination in the form $D+$ $\beta S_{t}$, with $\beta=(1-\alpha) /(1+\alpha)$ :

(i) If $\alpha \simeq 1$ (i.e., $\beta \simeq 0$ ), the roughness equally adjusts to $\partial u / \partial x$ and $\partial v / \partial y$. The roughness patterns represent divergence, and this is independent of the wind/ current angle. Nondivergent currents do not appear even if they are strained in one direction. The imaging mechanism, that is, the mechanism to detect currents from roughness variations, is thus unpolarized.

(ii) If $\alpha \ll 1$ (i.e., $\beta \simeq 1$ ), the roughness adjusts to $\partial u / \partial x$ and is insensitive to $\partial v / \partial y$. The imaging mechanism is completely polarized in the wind direction, that is, roughness patterns solely represent current gradients in the wind direction $\partial u / \partial x$. (iii) If $\alpha \gg 1$ (i.e., $\beta \simeq-1$ ), the imaging mechanism is completely polarized in the crosswind direction.

\section{b. Polarization and wave angular spread}

The polarization index mainly depends on the angular spread of the wave spectrum to be dependent on the wavenumber (e.g., Elfouhaily et al. 1997; Yurovskaya et al. 2013). The present subsection illustrates this by considering an idealized spectrum of background wind sea of the form

$$
\begin{aligned}
N_{0}(k, \phi) & \propto k^{m_{k}} \cos ^{n}(\phi) \text { for }-\pi / 2<\phi<\pi / 2 \\
& =0 \text { elsewhere, }
\end{aligned}
$$

where the wavenumber exponent $m_{k}$ is -4.5 for a saturation spectrum (Phillips 1958) and where the directionality cosine exponent $n$ takes typical values 

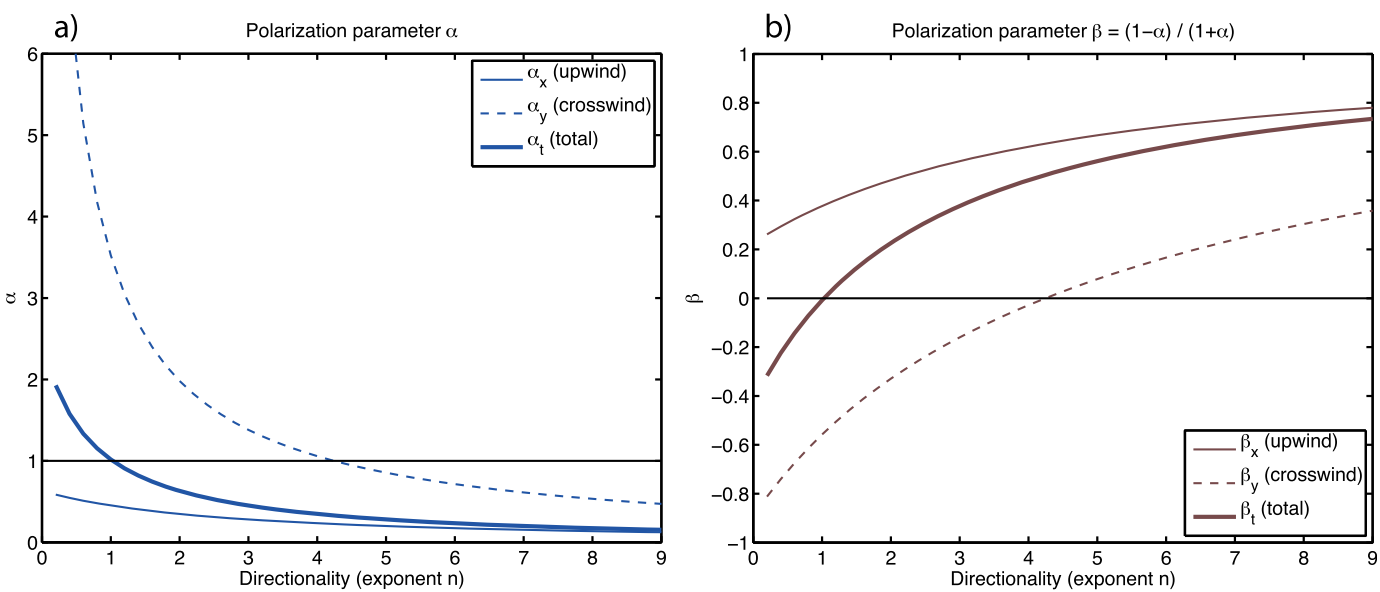

FIG. 3. (a) Polarization index $\alpha$ as a function of the wave spectrum directionality (the cosine exponent $n$ ). We use the idealized wave spectrum [(11-12)]. (b) Corresponding parameter $\beta=(1-\alpha) /(1+\alpha)$.

from around 1 for a broad spectrum (i.e., with widespread directions of wave propagations) to 9 for a narrow spectrum (i.e., with waves propagating mainly along the wind direction).

The angular spread of the relaxation time $\tau_{c}$ has to be prescribed. To do so we argue that, related to the growth rate parameters, waves traveling in the wind direction are more closely related to the local wind and adjust quicker than waves near the crosswind direction. For simplicity, the relaxation time is assumed to be separable, that is, $\tau_{c}(k, \phi)=\tau_{1}(k) \tau_{2}(\phi)$. By analogy with the relaxation time of Kudryavtsev et al. [2005, their (40)] we express

$$
\tau_{2}^{-1}(\phi)=\max [2|\cos (\phi)| \cos (\phi), 1] .
$$

Physically, (12) means that waves have a relaxation time independent of their direction except for waves within $45^{\circ}$ of the wind that have a shorter relaxation time.

Polarization parameters $\alpha_{x}, \alpha_{y}$, and $\alpha_{t}$ (for the upwind, crosswind, and total mss, respectively) are shown in Fig. 3a for our idealized wave spectrum [(11-12)], as a function of the directional spread. Prominent features are the following:

- Very directional wave spectra $(n \simeq 9)$ mainly adjust to $\partial u / \partial x$, with any $\alpha\left(\alpha_{x}, \alpha_{y}\right.$, or $\left.\alpha_{t}\right)$ of the order of $0.1-0.5$. This is expected as there are very few waves traveling in the crosswind direction $y$ in that case. On the contrary, less directional spectra (smaller $n$ ) are more sensitive to $\partial v / \partial y$.

- The upwind mss, as expected, mainly adjusts to $\partial u / \partial x$, with $\alpha_{x}$ of the order of 0.1-0.6 (thin solid line).
- The crosswind mss principally responds to $\partial u / \partial x$ for narrow spectra $(n \simeq 9)$, whereas for broad spectra $(n \simeq 1)$ it principally responds to $\partial v / \partial y$.

\section{c. Strain versus divergence signature}

Written in the form $D+\beta S_{t}$, with $\beta=(1-\alpha) /(1+\alpha)$, the polarization index gives information on the relative mss response to strain and divergence of similar amplitude. That is, a current divergence of magnitude $D$ (in $\mathrm{s}^{-1}$ ) produces an mss response similar to that of a current strain of magnitude $S_{t}=D / \beta$.

The polarization index $\beta$ is shown in Fig. $3 \mathrm{~b}$ from which two important points are made:

- The magnitude of $\beta$ is of the order of 0.5 , which means that the response to a current strain is smaller but has a similar order of magnitude as that of divergence. Quasigeostrophic surface currents have a small divergence compared to strain and are therefore expected to be observed due to their strain in the wind direction.

- Waves traveling in all directions become less steep when currents are divergent. On the contrary, when currents are strained in the wind direction, waves traveling in the wind direction become less steep, whereas waves traveling in the crosswind direction steepen. Under certain conditions (especially for crosswind-looking sensors), we thus expect $\beta$ to become negative, that is, we expect that the mss response to strain takes an opposite sign to that of the response to divergence. As shown in Fig. 3, such conditions occur when the wave spectrum is broad (e.g., $n<4$ ) for crosswind mss and when it is very broad (e.g., $n<$ 1 ) for the total mss. For upwind mss, such conditions never occur and $\beta$ is always positive. 

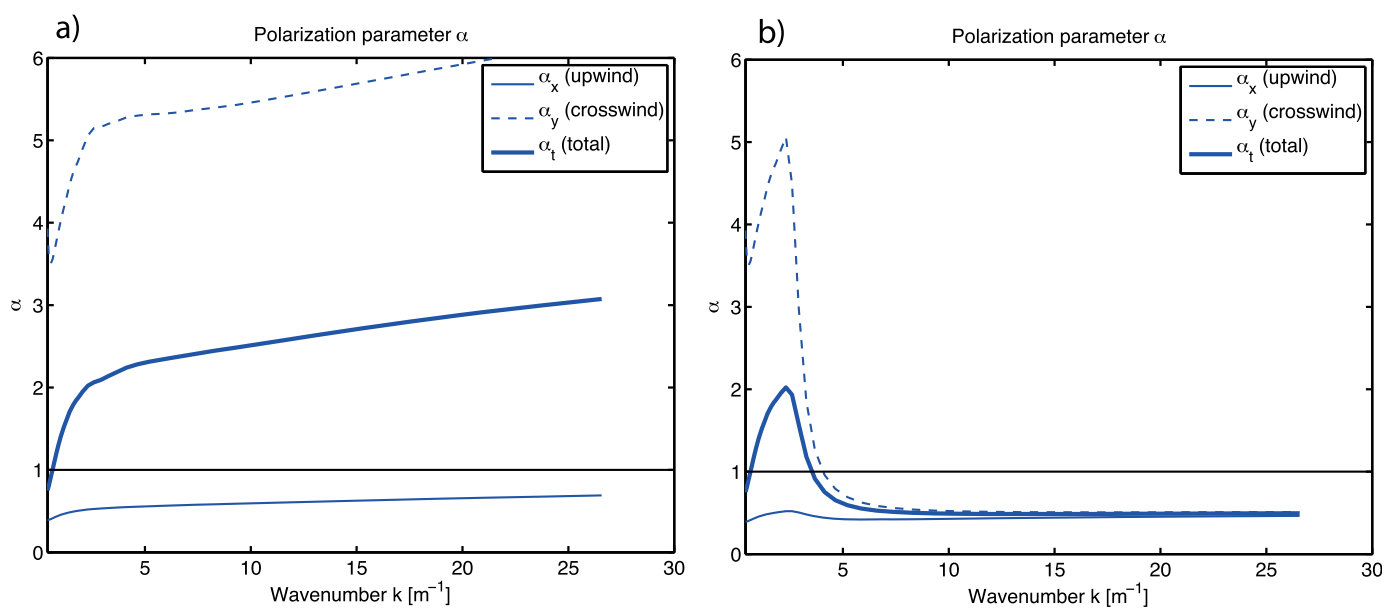

FIG. 4. Polarization index $\alpha$ as a function of the wavenumber $k$. We use a complete spectral shape of the wind sea as described in Kudryavtsev et al. (2005). Wind is set to $6 \mathrm{~m} \mathrm{~s}^{-1}$. (a) The creation of short waves by longer breaking waves is discarded, that is, we omit the term $Q^{w b}$ in Kudryavtsev et al. [2005, their (16)]. (b) Full model.

\section{d. Polarization for a complete spectrum}

For more realistic spectra, the energy-containing waves are quite directional, while smaller waves tend to be more omnidirectional (Donelan et al. 1985). In turn, short gravity waves with parasitic capillaries are often reported to gain directionality (Elfouhaily et al. 1997; Kudryavtsev et al. 1999). For our purpose, we use the background spectral form proposed by Kudryavtsev et al. (2005) constrained by recent observations (Yurovskaya et al. 2013). The polarization parameters $\alpha$ computed with this spectrum are shown in Fig. 4a. For most wavenumbers $\left(k>2 \mathrm{~m}^{-1}\right)$, the polarization corresponds to a case of broad spectrum $(n \simeq 1)$ in (11). The reason is that a $\cosh (\theta)^{-2}$ (Donelan et al.

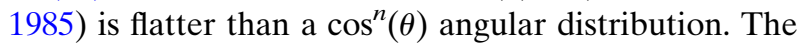
crosswind mss response is then always dominated by $\partial v / \partial y$ (Fig. 4a, dashed line) and thus the imaging mechanism for a crosswind-looking sensor is strongly polarized in the crosswind direction $\left(\alpha_{y}>1\right)$. On the contrary, the imaging mechanism for an upwind-looking sensor is solely polarized in the upwind direction $\left(\alpha_{x}<1\right)$.

\section{e. Generation of surface roughness by breaking waves}

Kudryavtsev et al. (2005) introduced an additional source term to take into account short-scale generation by longer breaking waves. The mechanism was shown to help in reproducing observed large variations of mss. Waves of intermediate scales, which are indeed experiencing the largest modulations in the presence of current, can further enhance the overall roughness through their breaking modulation.

Accounting for the effect of breaking waves on surface roughness leads to polarization parameters lower than 1 ( $\alpha \simeq 0.44$ ), for both upwind and crosswind mss (see Fig. 4b). Short waves created by larger, more directional breaking waves become indeed more sensitive to $\partial u / \partial x$. Note that polarization parameters for the upwind, crosswind, and total mss are then very similar for all short waves with $k>5 \mathrm{~m}^{-1}$.

\section{f. Practical summary}

The typical mss response to different current deformations is sketched on Fig. 2. To simplify, we assume that for the upwind (crosswind) mss, the imaging mechanism is polarized in the upwind (crosswind) direction.

Figure 2 is therefore not valid for the case corresponding to section $3 \mathrm{e}$ (for small waves, $k>5 \mathrm{~m}^{-1}$; see Fig. $4 \mathrm{~b}$ ), where for the crosswind mss the imaging mechanism is polarized in the upwind direction. In that case the crosswind mss would have the same response as that of the upwind mss.

In the special case of an unpolarized imaging mechanism, the responses to strain and shear would be zero.

\section{Illustration}

The polarization sensitivity is illustrated next with a numerical simulation of mesoscale and submesoscale oceanic turbulence in a beta-plane channel performed with a primitive equation model. The simulation is forced by an unstable westerly zonal flow. The turbulence is intensified at the surface where the submesoscale dynamics are active and the spectrum of horizontal kinetic energy is shallow (slope in $(2 \pi / \mathrm{L})^{-2}$ ). The averaged horizontal kinetic energy is about $10 \mathrm{~cm} \mathrm{~s}^{-1}$ at the surface. The horizontal resolution of the model is 

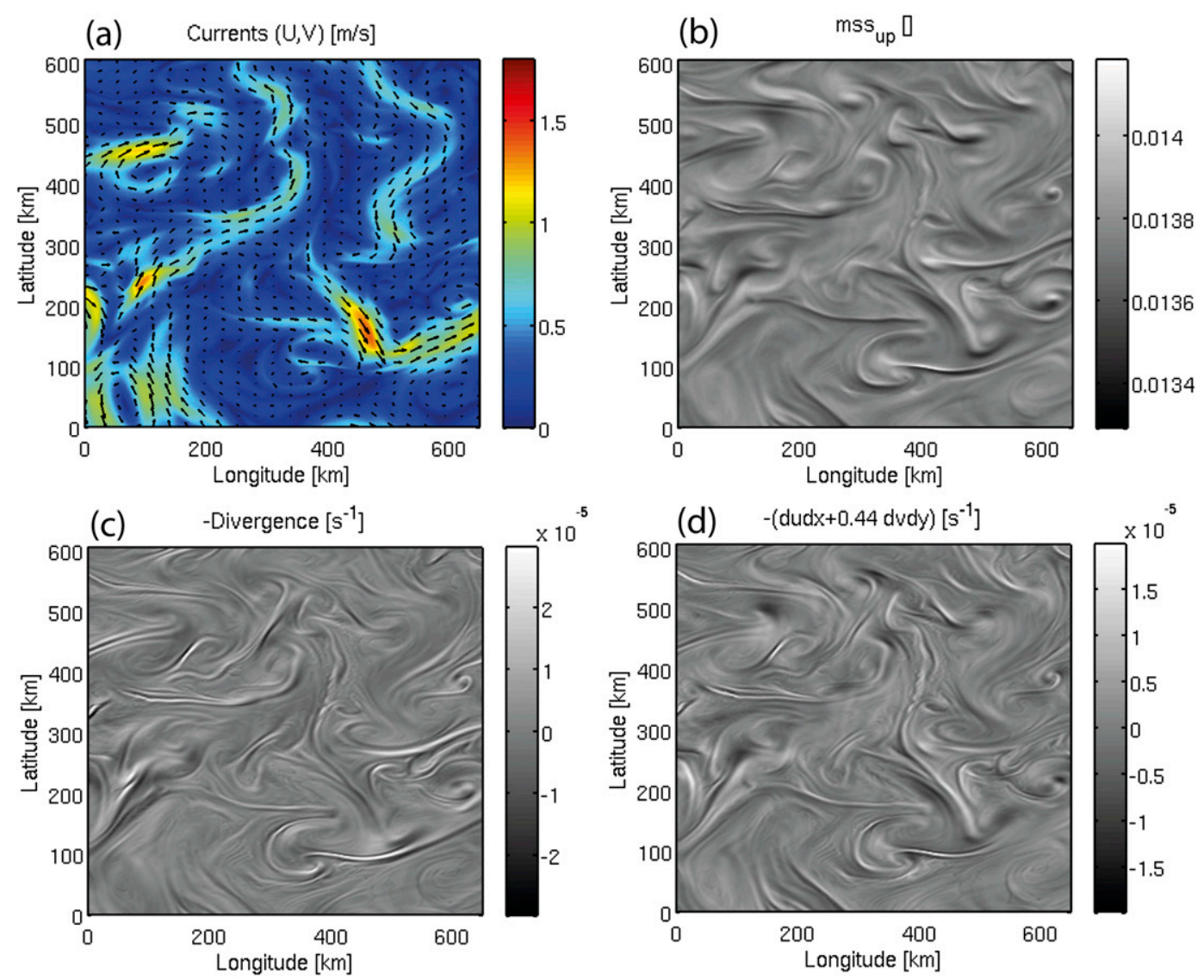

FIG. 5. (a) Surface current field of a numerical experiment of submesoscale turbulence. (b) Upwind mss response to those currents, simulated with the model of Kudryavtsev et al. (2005). The wind is westerly $6 \mathrm{~m} \mathrm{~s}^{-1}$ and background waves are supposed fully developed. (c) Surface current divergence. (d) $\partial u / \partial x+\alpha \partial u / \partial y$ with $\alpha=0.44$.

$2 \mathrm{~km}$, and the vertical resolution is about $3 \mathrm{~m}$ near the surface. More details can be found in Klein et al. (2008). Surface currents are shown in Fig. 5a.

The forward simulation of the mss variations uses the model of Kudryavtsev et al. (2005) and includes wave propagation [left-hand side of (1)]. The horizontal resolution of the wave model is also $2 \mathrm{~km}$. The wind is westerly $6 \mathrm{~m} \mathrm{~s}^{-1}$, and background waves are supposed fully developed. We consider here the mss produced by waves in the wavenumber range $5<k<28 \mathrm{~m}^{-1}$.

The resulting upwind mss is shown in Fig. 5b. The current convergence $(=-D)$ is shown in Fig. $5 \mathrm{c}$. It is not correlated to the upwind mss (corr $\simeq-0.08$ ). The average value of the polarization parameter $\alpha_{x}$ in this range of short waves is 0.44 (Fig. $4 \mathrm{~b}$, thin solid line), which corresponds to $\beta_{x}=0.39$. The field $-\partial u / \partial x-$ $0.44 \partial v / \partial y$ is shown in Fig. $5 \mathrm{~d}$. As can be visually checked, it is correlated with the upwind mss (corr $\simeq 0.56)$. The latter correlation is not perfect, but improves when the long-wave propagation is omitted (corr $>0.9$ ). This result validates the decomposition of the right-hand side of (1) with our polarization parameter.
Yet, even without propagation effects, the correlation between upwind mss and convergence remains very low (corr $\simeq 0.2$ ). The upwind mss anomaly $\widetilde{\mathrm{mss}}_{x}$ can thus be considered as a good tracer of $\partial u / \partial x+$ $0.44 \partial v / \partial y$. This field corresponds to $D+0.39 S_{t}$. In this submesoscale turbulence simulation, strain is roughly 3 to 4 times larger than divergence. Strain and divergence thus make equivalent contributions to the upwind mss anomaly and cannot be distinguished from a single image.

One can exploit properties of oceanic turbulence in order to advance further. Namely, the magnitude of divergence compared to that of strain varies with the horizontal scale $L$. In Fig. 6, we show the energy spectra of $D$ and of $0.39 S_{t}$. Strain contribution is larger at scales $L>25 \mathrm{~km}$, whereas divergence contribution is larger at scales $L<25 \mathrm{~km}$. For this particular oceanic turbulence, the upwind mss variations shall provide a reasonable source of information related to the divergence at high resolution $(L<25 \mathrm{~km})$ and to the strain in the wind direction at larger scales $(L>$ $25 \mathrm{~km})$. 


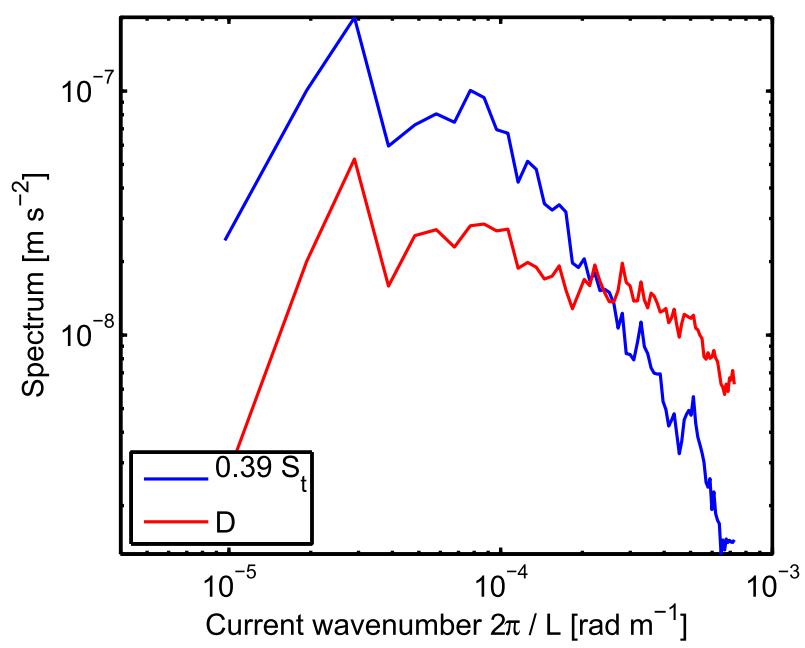

FIG. 6. Energy spectra of the divergence $D$ and weighted strain $0.39 S_{t}$ as a function of the horizontal scale of the current.

\section{Conclusions}

Surface wind waves interact with ocean currents to reveal specific deformation properties of surface currents. Considering surface roughness images, we limited our discussion to variations of wave mean square slope (mss). We ignored other mechanisms that potentially affect surface roughness, such as surfactants and atmospheric boundary layer modifications. Under a classical relaxation approach, the surface current gradient perturbs the background equilibrium sea state. We focused on waves of short and intermediate scales, where the propagation can be neglected compared to the current length scale. We also supposed that the wave spectrum is symmetrical about the wind direction.

Accordingly, the mss is modified by the along-wind current gradient $\partial u / \partial x$ or by the crosswind current gradient $\partial v / \partial y$. The other terms of the deformation field, $\partial u / \partial y$ and $\partial v / \partial x$, have no impact on the mss. This shows that currents with pure vorticity or with pure shear in the wind direction will not appear in roughness images.

The mss responses to $\partial u / \partial x$ and to $\partial v / \partial y$ are not equal in general, and their ratio defined a polarization parameter $\alpha$. If $\alpha \neq 1$, the imaging mechanism is called "polarized" in the sense that current detection depends on the relative direction between the wind and the current feature.

A convenient way to look at the imaging mechanism when polarized is through divergence and strain of the current. Divergent currents appear in any wind direction. On the contrary, only currents strained in the wind direction exhibit an mss variation, while currents strained $45^{\circ}$ to the wind do not appear. As such, the wind direction must be taken into account.
Additionally, the polarization parameter depends on whether the observation geometry favors upwind or crosswind components of the mss. Divergent (convergent) currents shall always appear as negative (positive) mss anomalies, while currents strained in the wind direction shall be sensor look (upwind or crosswind) dependent. This latter aspect further depends upon the directional spread of short wind waves and the interactions between short waves and longer breaking waves.

Thus, a very useful application of surface roughness images shall be to help diagnose upper-ocean vertical velocities due to submesoscale turbulence. As discussed, inversion of a single roughness image to obtain current divergence is certainly not straightforward. Especially when the imaging mechanism is polarized, the signature of the strain can be comparable in magnitude (around $30 \%)$ to that of divergence of similar amplitude. Yet, when using observations with very high resolution, the analysis can concentrate on horizontal scales for which divergence makes the dominant contribution. For larger scales, additional observations are needed to assess and possibly remove the strain signatures. The present analysis suggests the potential use of multiple observations with different azimuthal look angles of the sensor (for conditions and sensors for which the crosswind mss imaging is polarized in the crosswind direction) or multiple delayed observations with $45^{\circ}$ or $90^{\circ}$ different wind directions. The use of multiple look directions or possible acquisition with different wind directions can then help to retrieve surface currents from surface roughness observations.

Nevertheless, if individual images of surface roughness are to be used to characterize turbulence regimes in the upper ocean, a suitable diagnostic variable related to the wind direction, that is, $\partial u / \partial x+\alpha \partial v / \partial y$ including the polarization parameter $\alpha$, shall be used rather than the isotropic current divergence $\partial u / \partial x+\partial v / \partial y$.

Finally, in the perspective of future wide swath radar altimetry (e.g., the SWOT mission) for which the sensor physics is principally dominated by the mss strength, unique opportunities shall be offered to combine highresolution $(1 \mathrm{~km})$ detected surface roughness changes with sea surface elevation measurements at about $5-\mathrm{km}$ resolution.

Acknowledgments. We acknowledge the financial support of the French Agence Nationale pour la Recherche (ANR) through the REDHOTS project, of the European Space Agency (ESA) through the STSE MESO3D project and through the GlobCurrent project, and of the French Laboratoire d'Excellence (LabexMer) Axe1. We also acknowledge V. Kudryavtsev, RSHU, $\mathrm{SPb}$, for the fruitful discussions and help to implement the 
numerical forward simulation and F. Collard, OceanDataLab, to motivate the analysis and interpretation of numerous observations.

\section{REFERENCES}

Alpers, W., 1985: Theory of radar imaging of internal waves. $\mathrm{Na}$ ture, 314, 245-247, doi:10.1038/314245a0.

—, and I. Hennings, 1984: A theory of the imaging mechanism of underwater bottom topography by real and synthetic aperture radar. J. Geophys. Res., 89, 10529-10546, doi:10.1029/ JC089iC06p10529.

Apel, J. R., H. M. Byrne, J. R. Proni, and R. L. Charnell, 1975: Observations of oceanic internal and surface waves from the earth resources technology satellite. J. Geophys. Res., 80, 865881, doi:10.1029/JC080i006p00865.

Beal, R. C., P. S. DeLeonibus, and I. Katz, Eds., 1981: Spaceborne Synthetic Aperture Radar for Oceanography. Vol. 7. Johns Hopkins University Press, 215 pp.

, V. N. Kudryavtsev, D. R. Thompson, S. A. Grodsky, D. G. Tilley, V. A. Dulov, and H. C. Graber, 1997: The influence of the marine atmospheric boundary layer on ERS 1 synthetic aperture radar imagery of the Gulf Stream. J. Geophys. Res., 102, 5799-5814, doi:10.1029/96JC03109.

Cox, C., and W. Munk, 1954: Measurement of the roughness of the sea surface from photographs of the sun's glitter. J. Opt. Soc. Amer., 44, 838-850, doi:10.1364/JOSA.44.000838.

Donelan, M. A., J. Hamilton, and W. H. Hui, 1985: Directional spectra of wind-generated waves. Philos. Trans. Roy. Soc. London, A315, 509-562, doi:10.1098/rsta.1985.0054.

Dulov, V., and V. Kudryavtsev, 1990: Imagery of the inhomogeneities of currents on the ocean surface state. Sov. J. Phys. Oceanogr., 1, 325-336, doi:10.1007/BF02196830.

Elfouhaily, T., B. Chapron, K. Katsaros, and D. Vandemark, 1997: A unified directional spectrum for long and short wind-driven waves. J. Geophys. Res., 102, 15781-15796, doi:10.1029/ 97JC00467.

Espedal, H. A., O. M. Johannessen, J. A. Johannessen, E. Dano, D. Lyzenga, and J. Knulst, 1998: COASTWATCH'95: ERS 1/2 SAR detection of natural film on the ocean surface. J. Geophys. Res., 103, 24 969-24 982, doi:10.1029/98JC01660.

Ferrari, R., 2011: A frontal challenge for climate models. Science, 332, 316-317, doi:10.1126/science.1203632.

Fox-Kemper, B., and Coauthors, 2011: Parameterization of mixed layer eddies. III: Implementation and impact in global ocean climate simulations. Ocean Modell., 39, 61-78, doi:10.1016/ j.ocemod.2010.09.002.

Fu, L.-L., and B. Holt, 1983: Some examples of detection of oceanic mesoscale eddies by the SEASAT synthetic-aperture radar J. Geophys. Res., 88, 1844-1852, doi:10.1029/JC088iC03p01844.

—, D. Alsdorf, R. Morrow, E. Rodriguez, and N. Mognard, 2012: SWOT: The Surface Water and Ocean Topography mission: Wide-swath altimetric measurement of water elevation on Earth. Jet Propulsion Laboratory JPL Publ. 12-5, 228 pp. [Available online at http://hdl.handle.net/2014/41996.]

Hua, B. L., 1994: Skewness of the generalized centrifugal force divergence for a joint normal distribution of strain and vorticity components. Phys. Fluids, 6, 3200, doi:10.1063/1.868101

Hughes, B., 1978: The effect of internal waves on surface wind waves 2 . Theoretical analysis. J. Geophys. Res., 83, 455-465, doi:10.1029/JC083iC01p00455.

Huot, J., M. Rast, S. Delwart, J. Bezy, G. Levrini, and H. Tait, 2001: The optical imaging instruments and their applications:
AATSR and MERIS. ESA Bull., 106, 56-66. [Available online at http://esamultimedia.esa.int/multimedia/publications/ESABulletin-106/.]

Jansen, R. W., C. Y. Shen, S. R. Chubb, A. L. Cooper, and T. Evans, 1998: Subsurface, surface, and radar modeling of a Gulf Stream current convergence. J. Geophys. Res., 103, 18723-18743, doi:10.1029/98JC01195.

Johannessen, J. A., R. A. Shuchman, G. Digranes, D. Lyzenga, C. Wackerman, O. M. Johannessen, and P. Vachon, 1996: Coastal ocean fronts and eddies imaged with ERS 1 synthetic aperture radar. J. Geophys. Res., 101, 6651-6667, doi:10.1029/ 95JC02962.

, V. Kudryavtsev, D. Akimov, T. Eldevik, N. Winther, and B. Chapron, 2005: On radar imaging of current features: 2. Mesoscale eddy and current front detection. J. Geophys. Res., 110, C07017, doi:10.1029/2004JC002802.

Keller, W., and J. Wright, 1975: Microwave scattering and the straining of wind-generated waves. Radio Sci., 10, 139-147, doi:10.1029/RS010i002p00139.

Klein, P., B. Hua, G. Lapeyre, X. Capet, S. Le Gentil, and H. Sasaki, 2008: Upper ocean turbulence from high-resolution 3D simulations. J. Phys. Oceanogr., 38, 1748-1763, doi:10.1175/ 2007JPO3773.1.

Kudryavtsev, V. N., V. K. Makin, and B. Chapron, 1999: Coupled sea surface-atmosphere model: 2 . Spectrum of short wind waves. J. Geophys. Res., 104, 7625-7639, doi:10.1029/1999JC900005.

Kudryavtsev, V., D. Akimov, J. Johannessen, and B. Chapron, 2005: On radar imaging of current features: 1. Model and comparison with observations. J. Geophys. Res., 110, C07016, doi:10.1029/2004JC002505.

_ A. Myasoedov, B. Chapron, J. A. Johannessen, and F. Collard, 2012a: Joint sun-glitter and radar imagery of surface slicks. Remote Sens. Environ., 120, 123-132, doi:10.1016/ j.rse.2011.06.029.

,,,$--- \ldots$, and $\_, 2012 \mathrm{~b}$ : Imaging mesoscale upper ocean dynamics using synthetic aperture radar and optical data. J. Geophys. Res., 117, C04029, doi:10.1029/ 2011JC007492.

Kundu, P., 1990: Fluid Mechanics. Academic Press, 638 pp.

Lévy, M., R. Ferrari, P. J. Franks, A. P. Martin, and P. Rivière, 2012: Bringing physics to life at the submesoscale. Geophys. Res. Lett., 39, L14602, doi:10.1029/2012GL052756.

McWilliams, J., F. Colas, and M. Molemaker, 2009: Cold filamentary intensification and oceanic surface convergence lines. Geophys. Res. Lett., 36, L18602, doi:10.1029/2009GL039402.

Okubo, A., 1970: Horizontal dispersion of floatable particles in the vicinity of velocity singularities such as convergences. Deep-Sea Res. Oceanogr. Abstr., 17, 445-454, doi:10.1016/ 0011-7471(70)90059-8.

Phillips, O. M., 1958: The equilibrium range in the spectrum of wind-generated waves. J. Fluid Mech., 4, 426-433, doi:10.1017/ S0022112058000550.

- 1984: On the response of short ocean wave components at a fixed wavenumber to ocean current variations. J. Phys. Oceanogr., 14, 1425-1433, doi:10.1175/1520-0485(1984)014<1425: OTROSO $>2.0 . \mathrm{CO} ; 2$.

Plant, W. J., 1982: A relationship between wind stress and wave slope. J. Geophys. Res., 87, 1961-1967, doi:10.1029/ JC087iC03p01961.

Yurovskaya, M., V. Dulov, B. Chapron, and V. Kudryavtsev, 2013: Directional short wind wave spectra derived from the sea surface photography. J. Geophys. Res., 118, 4380-4394, doi:10.1002/ jgrc.20296. 